\title{
Interaction Design and Emotional Wellbeing
}

\section{David Coyle}

Computer Laboratory,

University of Cambridge,

Cambridge, UK, CB43EW

coyledt@tcd.ie

\section{Conor Linehan}

Lincoln Social Computing Research Centre,

University of Lincoln

Brayford Pool, UK LN6 7TS

clinehan@lincoln.ac.uk

\section{Karen P. Tang}

Department of Informatics

University of California, Irvine

Irvine, CA 92697 USA

kptang@ics.uci.edu

\section{Siân Lindley}

Socio-Digital Systems Group

Microsoft Research

Cambridge, UK, CB3 OFB

sianl@microsoft.com

Copyright is held by the author/owner(s).

CHI 2012, May 5-10, 2012, Austin, TX, USA.

ACM $x x x-x-x x x x-x x x x-x / x x / x x$.

\begin{abstract}
The World Health Organisation has concluded that emotional wellbeing is fundamental to our quality of life. It enables us to experience life as meaningful and is an essential component of social cohesion, peace and stability in the living environment [21]. This workshop will bring together a diverse community to consolidate existing knowledge and identify new opportunities for research on technologies designed to support emotional wellbeing. The workshop will examine uses of technology in mental health settings, but will also consider the importance of emotional needs in physical healthcare and wellbeing more generally. The design of technology to provide social support and to extend traditional care networks will be key workshop themes.
\end{abstract}

\section{Keywords}

Emotional wellbeing, health technologies, social support, storytelling, social and sensor networks, ethical requirements, privacy, theoretical frameworks

\section{ACM Classification Keywords}

H.5.2. User interfaces (User-centered design, evaluation/methodology), K.4.2 Social issues.

\section{General Terms}

Human Factors, Design

\section{Introduction}

Emotional wellbeing is a crosscutting concern that touches many aspects of our daily lives and has an impact across the lifespan. The World Federation for Mental Health defines emotional wellbeing as "a form of subjective well being, when individuals feel that they are coping, fairly in control of their lives, able to face 
challenges, and take on responsibility". Unfortunately, this definition does not describe the subjective or emotional state of a considerable proportion of people. Worldwide, for example, it is estimated that 151 million people suffer from depression at any one time, and 844,000 people die by suicide every year [9].

Studies have consistently shown that social support and interpersonal relationships can play a vital role in emotional wellbeing $[1,12]$. Conversely, social isolation is a major risk factor for mortality and is linked to higher rates of suicide and accidents, as well as a higher incidence of cardiovascular diseases and mental health conditions [12]. Thus, technologies that can effectively support social and emotional wellbeing offer significant benefits, both for individuals and society as a whole. This workshop will address questions such as:

1. What is the current state of the art in research on technology and emotional wellbeing?

2. How can new technologies, such as social and sensor networks, extend the scope of traditional mental healthcare services?

3. Can technology, in and of itself, provide social and emotional support?

4. How do we design for changing emotional needs across the lifespan?

5. What theoretical frameworks provide effective guidance for design and evaluation in this area?

6. What ethical and regulatory requirements should we consider when designing for emotional wellbeing?

7. In light of the potential benefits of technologymediated social support, how can we address possible privacy concerns?

8. What are the risks that technology can have a detrimental effect on emotional wellbeing?

\section{Tools and technologies}

Recent HCI research on emotional health has explored systems such as computerised CBT [6], virtual reality [6], relational agents [2], mobile systems [13] and computer games [7]. We will welcome papers offering new perspectives on each of these areas, but also invite papers addressing new possibilities, including:

Social networks: Outcome focused mental health research demonstrates that empowering relationships and the ability to draw on one's social network can play a vital role in successful health outcomes [1]. This raises many intriguing questions on the potential of social network technologies to support emotional wellbeing, some of which are beginning to be addressed $[3,15,17]$. For example, what are the characteristics of an effective emotional support network, and are they different from those of more general social networks, e.g. Facebook? Do we require new social networks that are purpose built to provide emotional support, or should we build within existing communities?

Digital storytelling: Many theories of emotional health, in particular Narrative Therapy [20], emphasise the importance of stories. Through narrative thinking we form a sense of self, a sense of the world around us and of our place in that world. Emotional health problems can arise when people tell overly negative stories or become blocked from telling their story, for reasons including social isolation, overly critical environments, purposeful oppression (as in the case of physical or emotional abuse, or ethical or political repression) or because a person feels their story simply does not fit with accepted or perceived social norms. Narrative therapy can be seen as a process of inviting people to tell and re-tell their life story, with the aim of reaching a more coherent and empowering narrative. We welcome submissions that consider digital 
storytelling, reminiscence, story sharing, and social identity construction to support emotional wellbeing.

\section{Technologies that provide emotional support:}

Given recent advances in areas such as affective computing and social robotics, we can begin to ask if technology can, in and of itself, provide social and emotional support. Examples to date include "Paro", a social robot for the elderly [19], and work on relational agents in clinical psychiatry [2]. The workshop will provide a forum to discuss the challenges, complexities and new experiences afforded by research in this area.

Monitoring emotional health: Traditionally, emotional health is monitored through (non-passive) self-report, often using paper-based diaries or behavioural questionnaires. Smart phones, contextaware sensing, and pervasive technology now offer increased opportunities, both for passive and nonpassive monitoring. A growing number of studies have examined the potential of systems to monitor and encourage behaviour change in non-health and physical healthcare domains [5]. Initial studies of non-passive monitoring of emotional health have also been undertaken [13]. Passive monitoring of emotional health presents many technical challenges, requiring data on thoughts and emotions, as well as behavioural data. It is however increasingly possible, at both an individual [16] and a societal level [10]. We can also consider the use of affect sensing to complement traditional self-monitoring techniques and help us to reflection more deeply upon our emotions [18].

\section{Challenges and theoretical frameworks}

Emotional health is a sensitive subject and the costs of design failures in this area are potentially severe. Health professionals are bound by a Hippocratic oath: often stated as "first do no harm". Alongside tools and technologies, this workshop will consider the ethical requirements and challenges associated with work in this area. In this abstract we highlight on one significant challenge - privacy - and also some of the diverse theoretical perspectives that can be brought to bear in reasoning about design in this area.

Privacy: Writing in the Annual Review of Clinical Psychology Hinshaw and Stier [11] report: "individuals with mental illness receive harsh stigmatization, resulting in decreased life opportunities and a loss of independent functioning over and above the impairments related to mental disorders themselves." Given such observations, it is unsurprising that details of emotional health are often closely guarded. Thus far we have highlighted the benefits of social interactions. However, when sharing potentially sensitive information, people may worry about how their disclosures will be received and interpreted. Thus, it is important that we consider privacy models and develop approaches that address the challenges of creating inclusive, emotionally supportive communities.

Theoretical frameworks and guidelines: Doherty et al. [8] have provided initial guidelines for the design of mental health technologies, but also highlight the need for further exploration and integration of the diverse theoretical frameworks in this area. This workshop will provide a forum for such a discussion. While systems implementing Cognitive Behavioural Therapy (CBT) have dominated early research on emotional health technologies [7], other perspectives, such as narrative and humanist theories or eclectic approaches such as the Skilled Helper Model, may prove equally valuable $[7,20]$. The integration of theoretical work from other disciplines may also serve as a catalyst for innovation. For example, "normalization process theory" [14] provides a framework for understanding how complex interventions can be implemented and integrated into 
everyday healthcare practices. Similarly, frameworks for the design and evaluation of complex interventions can help to guide rigorous and structured research [4].

\section{Workshop goals and themes}

HCI research on technology to support emotional health is in its early stages. This workshop will bring together a diverse community to share expertise and consolidate existing knowledge. It will provide a forum to define new opportunities, explore diverse theoretical frameworks, and set an agenda for future HCI research in this area. The role of social support and possibilities for extending traditional care networks will be core themes. The workshop will also explore the importance of emotional support in physical healthcare and wellbeing more generally, and will examine the requirements of technologies that can provide effective emotional support across the lifespan.

\section{Citations}

[1] Assay, T., et al., The Empirical Case for Common Factors in Therapy: Quantitative Findings, in The Heart and Soul of Change. 1999, APA: Washington. p.23-55.

[2] Bickmore, T., et al., Relational Agents in Clinical Psychiatry. Harvard Review of Psychiatry, 2010, 18(2).

[3] Burke, M., et al. Social network activity and social well-being. In proc ACM CHI 2010, p.1909-12.

[4] Campbell, M., et al., Framework for design and evaluation of complex interventions to improve health. British Medical Journal, 2000, 321 p.694-96.

[5] Consolvo, S., et al. Theory-driven design strategies for technologies that support behavior change in everyday life. In proc ACM CHI 2009, p.405-414.

[6] Coyle, D., et al., Computers in Talk-Based Mental Health Interventions. Interacting with Computers, 2007, 19(4) p.545-62.

[7] Coyle, D., et al. Exploratory evaluations of a computer game supporting cognitive behavioural therapy for adolescents. In proc ACM CHI 2011, p.2937-46.
[8] Doherty, G., et al., Design and evaluation guidelines for mental health technologies. Interacting with Computers, 2010, 22(4).

[9] Funk, M., et al., eds. Mental health and development: targeting people with mental health conditions as a vulnerable group. 2010, WHO: Geneva.

[10] Golder, S., et al., Diurnal and Seasonal Mood Vary with Work, Sleep, and Daylength Across Diverse Cultures. Science, 2011, 333(6051) p.1878-81.

[11] Hinshaw, S.P., et al., Stigma as Related to Mental Disorders. Annual Review of Clinical Psychology, 2008, 4 p.367-93.

[12] House, J.S., et al., Social relationships and health. Science, 1998, 241 p.540-45.

[13] Matthews, M., et al. In the mood: engaging teenagers in psychotherapy using mobile phones. In proc ACM CHI 2011, p.2947-56.

[14] May, C., et al., Implementing, Embedding, and Integrating Practices: An Outline of Normalization Process Theory. Sociology, 2009, 43 p.535-54.

[15] Newman, M.W., et al. "It's not that I don't have problems, I'm just not putting them on Facebook": Challenges and Opportunities in Using Online Social Networks for Health. In proc CSCW 20112011.

[16] Rachuri, K., et al. EmotionSense: A Mobile Phones based Adaptive Platform for Experimental Social Psychology Research. In proc UbiComp 2010.

[17] Skeels, M., et al. Catalyzing social support for breast cancer patients. In proc ACM CHI 2010, p.173-82.

[18] Ståhl, A., et al., Experiencing the Affective Diary. Personal and Ubiquitous Computing, 2009, 13(5).

[19] Wada, K., et al., Living With Seal Robots - Its sociopsychological and physiological influences on the elderly at a care house. IEEE Transactions on Robotics, 2007, 23(5) p.972-80.

[20] White, M., et al., Narrative means to therapeutic ends. 1990, New York: Norton.

[21] WHO. Mental Health Action Plan for Europe: Facing the Challenges, Building Solutions In proc WHO European Ministerial Conference on Mental Health 2005. 